\title{
Review of: "Landmark Alzheimer's drug approval confounds research community"
}

\author{
Jürgen Götz ${ }^{1}$ \\ 1 University of Queensland
}

Potential competing interests: The author(s) declared that no potential competing interests exist.

Jürgen Götz and Gerhard Leinenga:

We are watching the approval of Aducanumab with interest given that we had cloned the antibody to evaluate whether a combination with therapeutic ultrasound would be more effective in clearing amyloid and improving cognitive functions in the APP23 mouse strain. We had found that the combination therapy achieved up to 5-fold higher intracerebral antibody concentrations at the time point analysed. It also led to a lower amyloid burden and improved memory functions. Our data suggest that a combination therapy in humans may allow to reduce the antibody concentration required for achieving therapeutic outcomes.

Reference: Leinenga G, Koh WK, Götz」 (2021) A comparative study of the effects of Aducanumab and scanning ultrasound on amyloid plaques and behavior in the APP23 mouse model of Alzheimer disease, Alz Res Ther 13: 1-14 